\title{
Comparative Study of Impedance Eduction Methods, Part 2: NASA Tests and Methodology
}

\author{
M.G. Jones; W.R. Watson† B.M. Howerton ${ }^{\ddagger}$ \\ NASA Langley Research Center, Hampton, VA 23681 \\ and \\ S. Busse-Gerstengarbe ${ }^{\S}$ \\ Technische Universität Berlin, 10623 Berlin, Germany
}

\begin{abstract}
A number of methods have been developed at NASA Langley Research Center for eduction of the acoustic impedance of sound-absorbing liners mounted in the wall of a flow duct. This investigation uses methods based on the Pridmore-Brown and convected Helmholtz equations to study the acoustic behavior of a single-layer, conventional liner fabricated by the German Aerospace Center and tested in the NASA Langley Grazing Flow Impedance Tube. Two key assumptions are explored in this portion of the investigation. First, a comparison of results achieved with uniform-flow and shear-flow impedance eduction methods is considered. Also, an approach based on the Prony method is used to extend these methods from single-mode to multi-mode implementations. Finally, a detailed investigation into the effects of harmonic distortion on the educed impedance is performed, and the results are used to develop guidelines regarding acceptable levels of harmonic distortion.
\end{abstract}

\section{Introduction}

Over the last three decades, the NASA Langley liner physics team has investigated a number of methods to determine the acoustic impedance of sound-absorbing liners mounted in one wall of a flow duct. The majority of these methods use a duct propagation code coupled with acoustic pressure measurements acquired on the wall opposite the liner to educe the liner impedance. Some methods allow direct computation of the impedance based on the acoustic pressure profile (e.g., the single-mode method ${ }^{1}$ ), and others require implementation of an iterative approach to educe the impedance (e.g., a finite element method based on the convected Helmholtz equation ${ }^{2}$. A variety of numerical methods have been explored to improve the accuracy and efficiency of these impedance eduction methods. Although the majority of these investigations have been confined to the evaluation of methods implemented at NASA, comparative studies have proven invaluable in the extension of our understanding of liner physics. One such investigation was a joint effort between NASA Langley Research Center and three U.S. companies; General Electric Aircraft Engines, B. F. Goodrich and Boeing, ${ }^{3-5}$ in which similar liners were tested at each facility using their respective impedance eduction methods. This investigation demonstrated the value of using independent methods in a complementary manner, such that the liner impedance can be determined with increased confidence. It also provided valuable insights regarding advances that were needed in these impedance eduction methods.

This paper provides results of a recent comparative study conducted by the German Aerospace Center (DLR) and NASA. A single-layer liner was tested in the DLR DUCT-R (DUct aCoustic Test Rig - Rectangular cross section) and NASA Grazing Flow Impedance Tube (GFIT), such that a direct comparison of the respective impedance eduction methods could be conducted. This liner consists of an aluminum perforated

\footnotetext{
* Senior Research Scientist, Research Directorate, Structural Acoustics Branch, Associate Fellow AIAA.

${ }^{\dagger}$ Senior Research Scientist, Research Directorate, Computational AeroSciences Branch, Associate Fellow AIAA.

${ }^{\ddagger}$ Research Scientist, Research Directorate, Structural Acoustics Branch, Senior Member AIAA.

$\S$ Research Assistant, Institute of Fluid Mechanics and Engineering Acoustics, Student Member AIAA
} 
facesheet that is mounted onto a core consisting of cylindrical channels aligned with the facesheet holes (one hole per channel). These channels are terminated with a rigid back plate.

The results of this comparative study are presented in two papers. The first ${ }^{6}$ describes the DUCT-R flow duct, the acoustic liner, and the DLR method for impedance eduction. That paper provides results of the tests conducted in the DUCT-R. Also, previously acquired NASA GFIT data are analyzed with the DLR eduction method, and the results are compared with published NASA results.

The current paper provides results acquired with the GFIT at four Mach numbers, four source sound pressure levels, and forty frequencies. The resultant database is used for a detailed investigation of the test liner with multiple impedance eduction methods. The impedance eduction methods are presented in Section II and the experimental approach is discussed in Section III. Key results and concluding remarks are presented in Sections IV and V, respectively.

\section{Impedance Eduction Methods}

In the following, five impedance eduction methods are presented. The Pridmore-Brown equation is used as the basis for the first four methods. The last method is the finite element method based on the convected Helmholtz equation that has been the predominant method in use at NASA for almost two decades. Figure 1 provides a schematic of the three-dimensional flow duct and Cartesian coordinate system used with these impedance eduction methods. The origin is located in the lower left corner of the duct, facing downstream. The surface of the liner with uniform, normalized impedance, $\zeta$, comprises a portion of the upper wall of the flow duct (i.e., $y=H$ ) over the axial extent of $L_{1} \leq x \leq L_{2}$. (All impedances presented in this paper are normalized with the characteristic impedance, $\rho c$, of the air flowing in the duct.) All other walls of the duct are assumed rigid, and the source and exit planes of the computational domain are located at $x=0$ and $x=L$, respectively. The sound source is assumed to be a single tone at a frequency such that only zero-order horizontal modes are present, thereby allowing the analysis to be restricted to the $(x, y)$-plane.

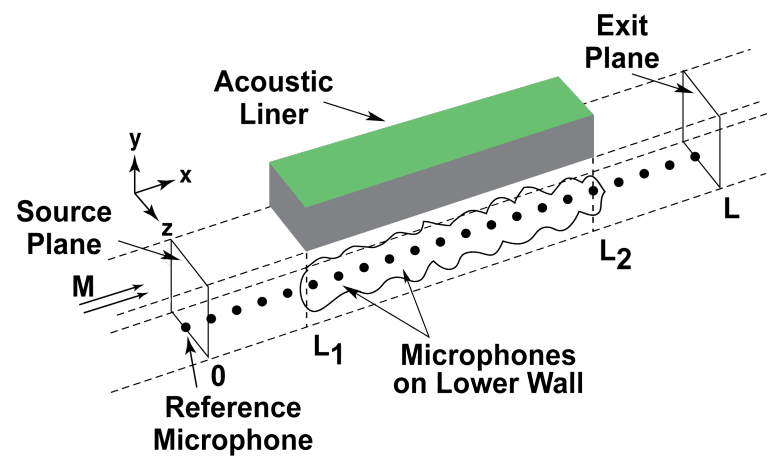

Figure 1: Sketch of Grazing Flow Impedance Tube computational domain.

\section{A. Methods Based on the Pridmore-Brown Equation}

The first four impedance eduction methods are based on numerical solution of the Pridmore-Brown equation. ${ }^{7}$ Two methods assume a single, unidirectional mode is dominant over the axial extent of the liner, and the other two methods relax this constraint to allow for multiple modes and reflections in the lined region. All four methods assume the side walls are rigid, and that only zero-order modes are present in the spanwise dimension (perpendicular to side walls). 


\section{Single-Mode Methods}

The Pridmore-Brown equation is

$$
\frac{d^{2} P}{d y^{2}}+\frac{2 k_{x}}{\left(k-M k_{x}\right)} \frac{d M}{d y} \frac{d P}{d y}+\left[k^{2}-2 k k_{x} M-\left(1-M^{2}\right) k_{x}^{2}\right] P=0
$$

where $k$ is the free-space wavenumber, $k_{x}$ is the axial propagation constant, and $M$ is the mean flow Mach number. The 1-D normal mode, $P$, is provided by the relation

$$
p=P(y) e^{-i k_{x} x}
$$

where an $e^{i \omega t}$ time dependence is assumed and $p$ is the acoustic pressure. Along the lower wall, the rigid wall condition gives

$$
\frac{d P}{d y}=0 \quad \text { at } y=0
$$

and along the upper wall, $p$ must satisfy the locally-reacting wall impedance boundary condition presented by Myers. ${ }^{8}$ Thus, the normal mode, $P$, must satisfy

$$
\frac{d P}{d y}=\frac{-i k P}{\zeta}\left(1-\frac{k_{x}}{k} M\right)^{2} \quad \text { at } y=H
$$

Equation (1) is solved using a shooting point method, with the boundary conditions at the lower rigid wall given as

$$
P(0)=1 \quad \text { and }\left.\quad \frac{d P}{d y}\right|_{y=0}=0
$$

Given $P(0), \frac{d P(0)}{d y}$ and $k_{x}$, the shooting point method is used to integrate the Pridmore-Brown equation to the upper wall (assumed located at $y=H$ ) to obtain $P(H)$ and $\frac{d P(H)}{d y}$.

The axial propagation constant, $k_{x}$, is computed from a measurement of the sound pressure level and phase decay rates, $\frac{d \mathrm{SPL}}{d x}$ and $\frac{d \phi}{d x}$, using the equation:

$$
k_{x}=-\frac{d \phi}{d x}+\frac{d \mathrm{SPL}}{d x} \frac{i}{20 \log _{10}(e)}
$$

This assumes that the axial sound pressure level and phase decay rates can be determined from the acoustic pressures measured on the wall opposite the liner (i.e., $y=0$ ), and that they are negative for right-moving waves. It also assumes that this acoustic pressure profile is dominated by a single, unidirectional mode.

Clearly, the acoustic liner is not infinitely long, as assumed in this model. In practice, this infinitelength assumption is satisfied if the liner length $\left(L_{2}-L_{1}\right.$ in Fig. 1$)$ is sufficiently large that reflections and near-field effects generated at the liner leading and trailing edges are suppressed over the majority of the liner axial extent. For the current investigation, a portion of the acoustic pressure profile (as measured along the lower wall opposite the liner) that extends over the $457 \mathrm{~mm}$ axially centered portion of the liner $\left(L_{1}+51.8 \mathrm{~mm}<x<L_{2}-51.8 \mathrm{~mm}\right)$ is used to determine the SPL and phase decay rates (see Fig. 2). The selected portion of the acoustic pressure profile is curve-fit (linear slope) to compute the required decay rates, and correlation coefficients are computed for these curve-fits to form the basis for determining whether a single mode is dominant over the axial extent of interest. For those test conditions where the correlation coefficients are sufficiently high to suggest the acoustic pressure profile is dominated by a single mode (i.e., SPL and $\phi$ profiles decay with constant slopes), the acoustic liner surface impedance is then computed from Eq. (4) to obtain 


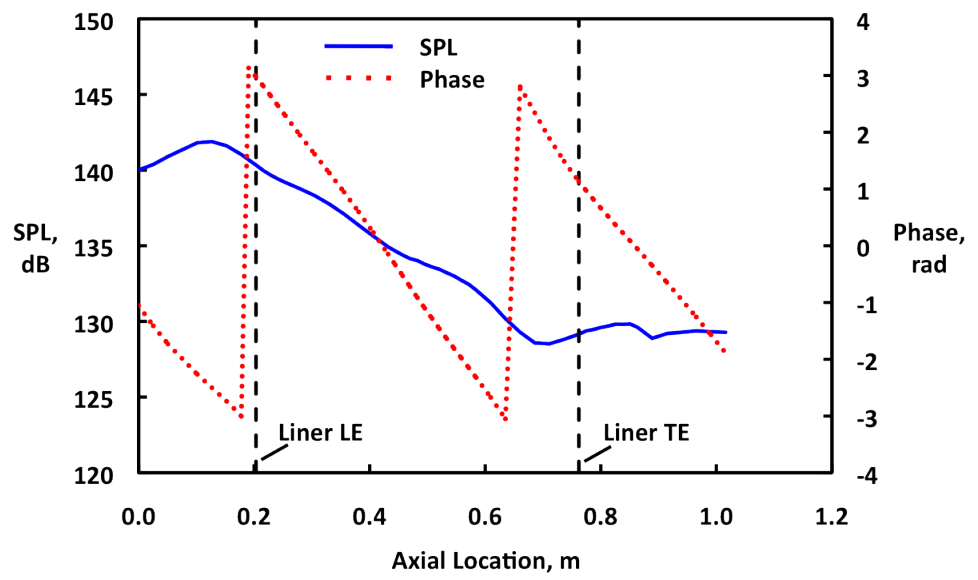

Figure 2: Sample SPL \& phase profiles.

$$
\zeta=-i k P\left(1-\frac{k_{x}}{k} M\right)^{2}\left(\frac{d P}{d y}\right)^{-1} \quad \text { at } y=H
$$

It should be noted that this model makes no specific assumption regarding the mean flow profile; i.e., it holds for uniform flow $(M(y)=$ constant $)$ or 1-D shear flow $(M(y) \neq$ constant). For convenience, the uniform and shear-flow versions of this model will be referenced as PBU-SM (Pridmore-Brown, uniform flow, single-mode method) and PBS-SM (Pridmore-Brown, 1-D shear flow, single-mode method), respectively.

\section{Multi-Mode Methods}

The single-mode assumption is satisfied for the majority of data acquired in the GFIT. Nevertheless, it is not uncommon to encounter conditions for which multiple modes or reflections are evident in the complex pressure profile measured on the wall opposite the liner. For these occasions, Prony's method ${ }^{9}$ is used to extract a single axial wavenumber, $k_{x}$, from the multi-modal sound field. In the current implementation, complex acoustic pressures are measured using $2 m$ equally spaced microphones flush-mounted in the wall opposite the liner ( $m=6$ for the current investigation). These data are then used to solve the matrix equation

$$
[C]\{Y\}=-\{P\}
$$

via a complex linear equation solver, where

$$
[C]=\left[\begin{array}{cccccc}
p_{0} & p_{1} & \cdots & p_{m-3} & p_{m-2} & p_{m-1} \\
p_{1} & p_{2} & \cdots & p_{m-2} & p_{m-1} & p_{m} \\
p_{2} & p_{3} & \cdots & p_{m-1} & p_{m} & p_{m+1} \\
p_{3} & p_{4} & \cdots & p_{m} & p_{m+1} & p_{m+2} \\
\vdots & \vdots & \vdots & \vdots & \vdots & \vdots \\
p_{m-1} & p_{m} & \cdots & p_{2 m-4} & p_{2 m-3} & p_{2 m-2}
\end{array}\right],\{Y\}=\left\{\begin{array}{c}
D_{1} \\
D_{2} \\
D_{3} \\
D_{4} \\
\vdots \\
D_{m}
\end{array}\right\},\{P\}=\left\{\begin{array}{c}
p_{m} \\
p_{m+1} \\
p_{m+2} \\
p_{m+3} \\
\vdots \\
p_{2 m-1}
\end{array}\right\}
$$

and $p_{j}$ represents the complex acoustic pressure at location $j(0 \leq j \leq 2 m-1)$.

The resultant values of $D_{J}(1 \leq J \leq m)$ are used to create the polynomial

$$
F(X)=D_{1}+D_{2} X+D_{3} X^{2}+D_{4} X^{3}+\cdots+D_{m} X^{m-1}+X^{m}
$$

and the complex roots, $X_{n}$, of this polynomial are used to determine the axial wavenumber, 


$$
k_{x}=\frac{-i \log _{e}\left(X_{n}\right)}{\Delta x}
$$

The axial wavenumber is then used in Eq. (7) to determine the liner impedance. The uniform and shear-flow versions of this model will be referenced as PBU-MM (Pridmore-Brown, uniform flow, multi-mode method) and PBS-MM (Pridmore-Brown, 1-D shear flow, multi-mode method), respectively.

\section{B. Method Based on the Convected Helmholtz Equation}

An impedance eduction method based on the convected Helmholtz equation (CHE) is also used for the current investigation. The underlying model has been presented previously, ${ }^{10}$ and is summarized here for the sake of convenience.

Throughout the flow duct, the acoustic pressure field, $p$, is assumed to satisfy the convected Helmholtz equation

$$
\left(1-M^{2}\right) \frac{\partial^{2} p}{\partial x^{2}}+\frac{\partial^{2} p}{\partial y^{2}}-2 i k M \frac{\partial p}{\partial x}+k^{2} p=0
$$

Mean flow profiles measured just upstream and downstream of the computational domain are used to compute average axial flow velocities, and a linear fit between the two axial locations is used to determine the uniformflow axial Mach number, $M$. The liner is assumed to be locally-reacting, and to be properly represented by the Myers $^{8}$ wall impedance boundary condition,

$$
-\frac{\partial p}{\partial y}=i k\left(\frac{p}{\zeta_{u}}\right)+2 M \frac{\partial}{\partial x}\left(\frac{p}{\zeta_{u}}\right)+\frac{M^{2}}{i k} \frac{\partial^{2}}{\partial x^{2}}\left(\frac{p}{\zeta_{u}}\right)
$$

where $\zeta_{u}$ represents the impedance of the upper wall and the curvature terms are neglected for a flow duct with straight walls (e.g., the GFIT). The admittance (i.e., $1 / \zeta_{u}$ ) is taken as zero for rigid upper wall sections of the duct located upstream and downstream of the liner, and $\zeta_{u}=\zeta$ is used in the liner portion of duct. The boundary condition of the rigid lower wall is

$$
\frac{\partial p}{\partial y}=0
$$

For a source consisting solely of the zero-order mode, the acoustic pressure profile at the source plane, $p(0, y)$, is assumed constant, and is taken to be equal to the acoustic pressure measured at the reference microphone on the lower wall, $p(0,0)$. This can be expressed as the source plane boundary condition

$$
p(0, y)=p(0,0)
$$

Similarly, the exit plane acoustic pressure boundary condition is based on the acoustic pressure measured by the microphone on the lower wall at $x=L$, and is expressed as

$$
p(L, y)=p(L, 0)
$$

Since the impedance depends upon the axial location, Eqs. (12)-(16) do not have exact solutions, except for very limited cases. Consequently, they are solved numerically using a continuous Galerkin finite element method. A general purpose numerical procedure is used to determine the impedance that reproduces the acoustic pressure field measured on the wall opposite the test liner. The unknown liner impedance, $\zeta$, is selected such that the objective function

$$
F(\zeta)=\sum_{I=1}^{n \text { wall }}\left\{\left.p\left(x_{I}, 0\right)\right|_{\mathrm{FEM}}-\left.p\left(x_{I}, 0\right)\right|_{\text {Meas }}\right\}\left\{\left.p^{*}\left(x_{I}, 0\right)\right|_{\mathrm{FEM}}-\left.p^{*}\left(x_{I}, 0\right)\right|_{\text {Meas }}\right\}
$$

is minimized, where the superscript ${ }^{*}$ represents the complex conjugate. This objective function may be interpreted as the difference between the measured, $\left.p\left(x_{I}, 0\right)\right|_{\text {Meas }}$, and numerically computed, $\left.p\left(x_{I}, 0\right)\right|_{\mathrm{FEM}}$, wall pressures opposite the test liner at a number, nwall, of microphone locations over the range of $0 \leq x \leq L$. 
The objective function is minimized using a gradient-based optimizer (i.e., the Davidon-Fletcher-Powell optimization technique), in which numerical differentiation is used to compute the gradient of $F(\zeta)$.

An initial guess of the liner impedance is required for the optimization technique. For the current investigation, four independent optimizations are conducted for each test condition, each with a unique starting value of the liner impedance. These starting values are $0.5+2 i, 0.5-2 i, 2+2 i$, and $2-2 i$. A comparison of the four resultant educed impedances is used to determine whether any of the results are due to local optima. If all four values are identical, the result is generally assumed to be a global optimum (i.e., the correct impedance). If the four values are not identical, physical reasoning is applied to determine which ones are anomalous.

\section{Experimental Approach}

The NASA grazing flow impedance tube (GFIT) has a cross-sectional geometry of $50.8 \mathrm{~mm}$ wide by $63.5 \mathrm{~mm}$ high, such that higher-order modes in the horizontal and vertical dimensions cut on at different frequencies. It allows convenient evaluation of acoustic liners with lengths from $50.8 \mathrm{~mm}$ to $609.6 \mathrm{~mm}$. As depicted in Fig. 3, flow propagates from left to right. High pressure air is supplied on the upstream end, and a vacuum blower is located on the downstream end, such that the test window containing the acoustic liner is exposed to near-ambient pressure conditions for mean flows up to Mach 0.6. The surface of the test liner forms a portion (533.4 $\mathrm{mm}$ for the current investigation) of the upper wall of the flow duct. The source section consists of up to eighteen acoustic drivers, which can be mounted upstream (exhaust mode, see Fig. 3) or downstream (inlet mode) of the test section, and can be used to generate tones (one frequency at a time) at up to $150 \mathrm{~dB}$ over a frequency range of 400 to $3000 \mathrm{~Hz}$.

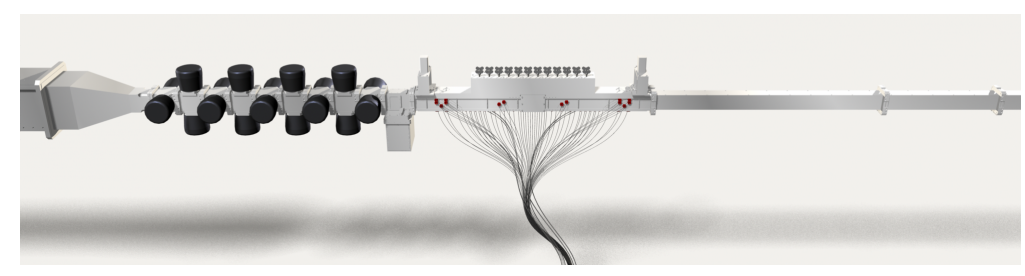

Figure 3: Sketch of Grazing Flow Impedance Tube.

Fifty-three microphones flush-mounted in the lower wall (opposite the liner) are used to measure the acoustic pressure field over the axial extent of $0 \leq x \leq L$ (see Fig. 1), such that the impedance eduction methods described in Section II can be used to determine the impedance of the test liner. For each data acquisition, 1000 averages on each microphone channel (blocks of 1024 data points per average) are taken. To reduce the influence of flow noise, a cross-spectrum signal extraction method ${ }^{11}$ is used to determine the amplitudes and phases at each of the microphone locations relative to the amplitude and phase at the reference microphone location.

A dual-axis traverse probe system is used to measure the flow profile at selected axial planes in the GFIT. Two total-pressure pitot probes are used for this measurement. One is shaped to allow detailed measurements close to the upper wall, and the other is shaped to allow corresponding measurements near the lower wall. The traverse system uses two stepping motors to successively move the pitot probe to preselected measurement locations, such that the full flow profile can be accurately determined. This system is used to acquire flow profiles upstream and downstream of the liner, from which average flow Mach numbers are computed. The traverse probe is removed for subsequent acoustic measurements.

A conventional, single-layer liner (Fig. 4) was chosen for this comparative study. The perforate facesheet has a thickness of $1 \mathrm{~mm}$, a hole diameter of $1 \mathrm{~mm}$, and a porosity of $1.5 \%$. This facesheet is mounted onto a $40 \mathrm{~mm}$-deep core consisting of $6.9 \mathrm{~mm}$-wide cylindrical chambers, and the liner back plate is rigid. The liner treatment is $533.4 \mathrm{~mm}$ long, and is mounted in a $558.2 \mathrm{~mm}$ long frame. More details regarding the liner are provided in the companion paper. ${ }^{6}$ 


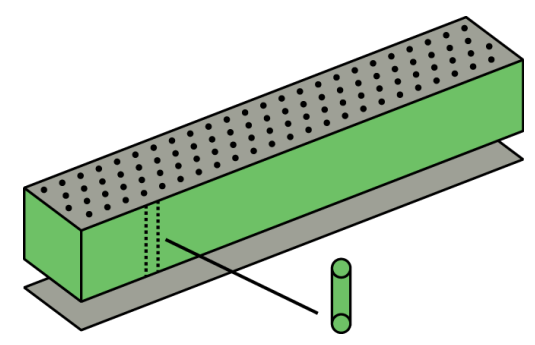

Figure 4: Sketch of single-layer liner.

For the current investigation, the acoustic liner was tested with an upstream source (exhaust mode), as depicted in Fig. 3. The GFIT acoustic driver array produced a tonal source (one frequency at a time), at frequencies of 410 to $2750 \mathrm{~Hz}$ in $60 \mathrm{~Hz}$ increments. Acoustic measurements were acquired using the microphone array for each of these frequencies, at source sound pressure levels of 110, 120, 130 and $140 \mathrm{~dB}$, and at centerline Mach numbers of 0.0, 0.1, 0.2 and 0.3 .

\section{Results}

Five distinct impedance eduction methods were discussed in Section II. The first four are based on the Pridmore-Brown equation, two that assume a single, unidirectional mode is dominant over the axial extent of the liner, and two that allow for multiple modes and reflections to be dominant in this section of duct. These methods are labeled as PBU-SM, PBS-SM, PBU-MM and PBS-MM, where "PBU" and "PBS" represent the Pridmore-Brown method with uniform and shear flow, respectively, and the "SM" and "-MM" suffixes indicate whether only one (single-mode) or more (multi-mode) modes are being considered in the lined region of the duct. The last method is based on the convected Helmholtz equation. This method assumes uniform flow, and that only zero-order modes are present at the source and termination planes of the computational domain.

As discussed in Section III, numerous test conditions are evaluated. This allows exploration of multiple liner impedance features, some more obvious than others. Results are presented to demonstrate comparisons of each of the methods presented in Section II. The methods based on the uniform-flow assumption compare favorably, as do the corresponding methods based on the shear-flow assumption. However, the differences between the results educed with the uniform-flow and shear-flow methods are markedly different. Nearly all of the measured acoustic pressure data contain very linear amplitude and phase decays along the axial extent of the liner (see Fig. 2). This is a clear indication that a single mode is dominant in the lined region. For this reason, the PBU-SM method is used as the baseline method for the remainder of this investigation.
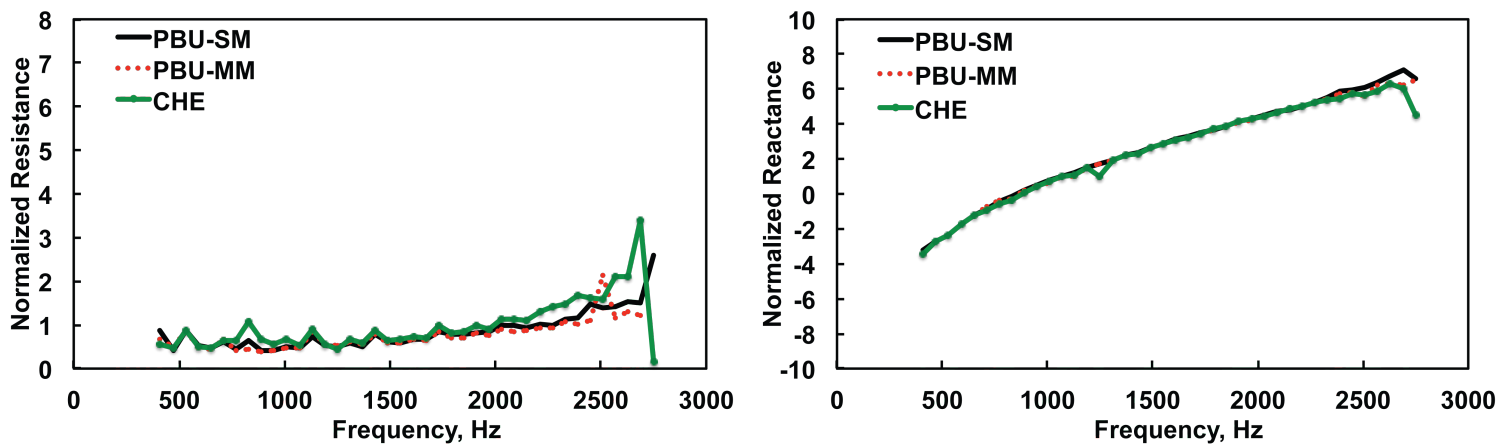

Figure 5: Comparison of three uniform-flow methods for impedance eduction; Mach 0.0, source SPL of $120 \mathrm{~dB}$. 


\section{A. Comparison of Impedance Eduction Methods}

Figure 5 provides a comparison of impedance spectra educed with each of the uniform-flow eduction methods. These results were acquired at Mach 0.0, with the source SPL set to $120 \mathrm{~dB}$. In general, the results compare quite favorably. At the highest frequency $(2750 \mathrm{~Hz})$, the first higher-order vertical mode is cut on in the GFIT. This violates the assumptions underlying the CHE. Otherwise, the resistances educed with the CHE at the upper end of the frequency range are above those educed with the PBU-SM and PBU-MM. Based on the corresponding reactance spectrum, this frequency regime is near anti-resonance for this liner, which tends to be a sensitive area for impedance eduction with the CHE.

Figure 6 provides the corresponding results based on data acquired at Mach 0.3, with the source SPL set to $120 \mathrm{~dB}$. The CHE results are observed to track the PBU-SM results across much of the frequency range, with deviations at the low and high frequency extremes. The CHE has been previously reported to have difficulty at the lowest frequencies (tends to give slightly higher resistance values than expected). ${ }^{12}$ One possible cause for this low-frequency difficulty is the length of the liner, i.e., perhaps the liner is not "exposed" to a sufficient portion of the test frequency wavelength to support the impedance eduction process. The highest frequencies are above cut-on of the first higher-order mode, and therefore violate the underlying assumption used in the CHE (see Eqns. (15 and 16)).

At Mach 0.0, the single-mode and multi-mode methods based on the Pridmore-Brown equation (PBU-SM and PBU-MM) compare very well. This comparison degrades somewhat at Mach 0.3 , most notably at the lowest frequencies. This may be related to the manner in which the Prony method is used to educe the axial wavenumbers of the first six modes. For example, additional constraints may be needed in the automated process used to select the propagating mode from those computed using the Prony method. Further analysis is needed to more thoroughly investigate these issues.
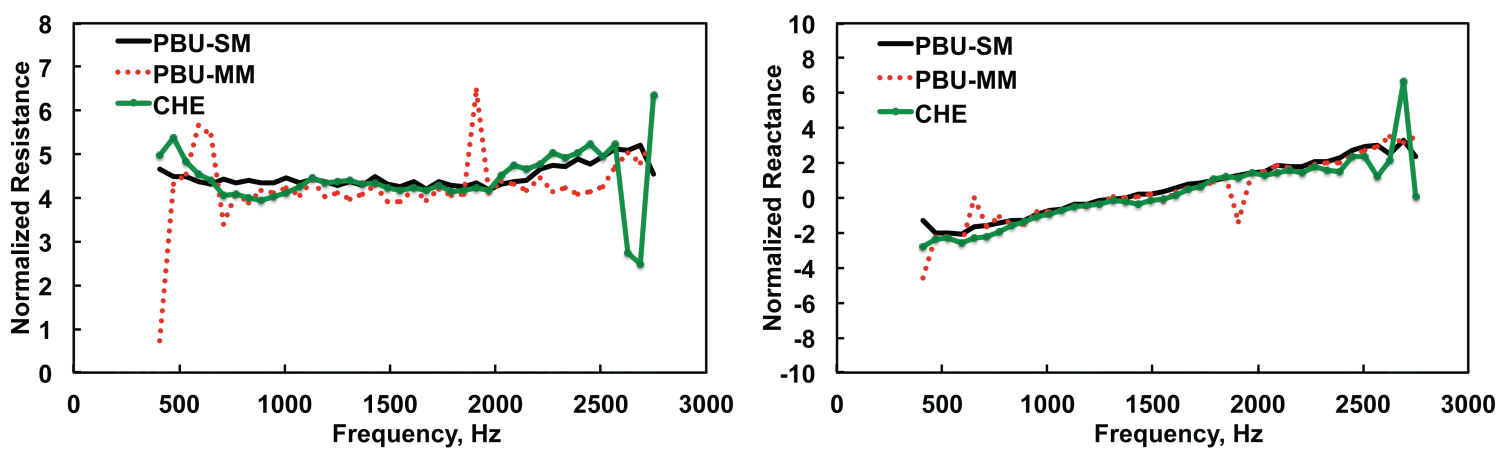

Figure 6: Comparison of three uniform-flow methods for impedance eduction; Mach 0.3, source SPL of $120 \mathrm{~dB}$.

Figure 7 provides a comparison of impedance spectra educed using the uniform-flow and shear-flow versions of the Pridmore-Brown methods. These results are educed from data acquired at Mach 0.3, with a source SPL of $120 \mathrm{~dB}$. A number of features are evident in these results. First, the two shear-flow methods (PBS-SM and PBS-MM) compare favorably at all but the lowest frequencies. Given the results mentioned earlier regarding this frequency range, this suggests that the longer wavelengths at these frequencies are problematic for many of the current eduction methods. Further investigation into this area is needed. The more dominant features evident in Fig. 7 are the shapes of the resistance and reactance spectra. The resistance spectrum has a near-zero slope for the uniform-flow methods, as compared to a large negative slope for the shear-flow methods. The low-frequency resistance educed with shear-flow methods is increased relative to those educed with uniform-flow methods, and the opposite holds for the higher frequencies. In addition, the shape of the reactance spectrum is changed from a $-\cot (k d)$ pattern $(d$ is the cavity depth of the liner) to a relatively flat curve. 

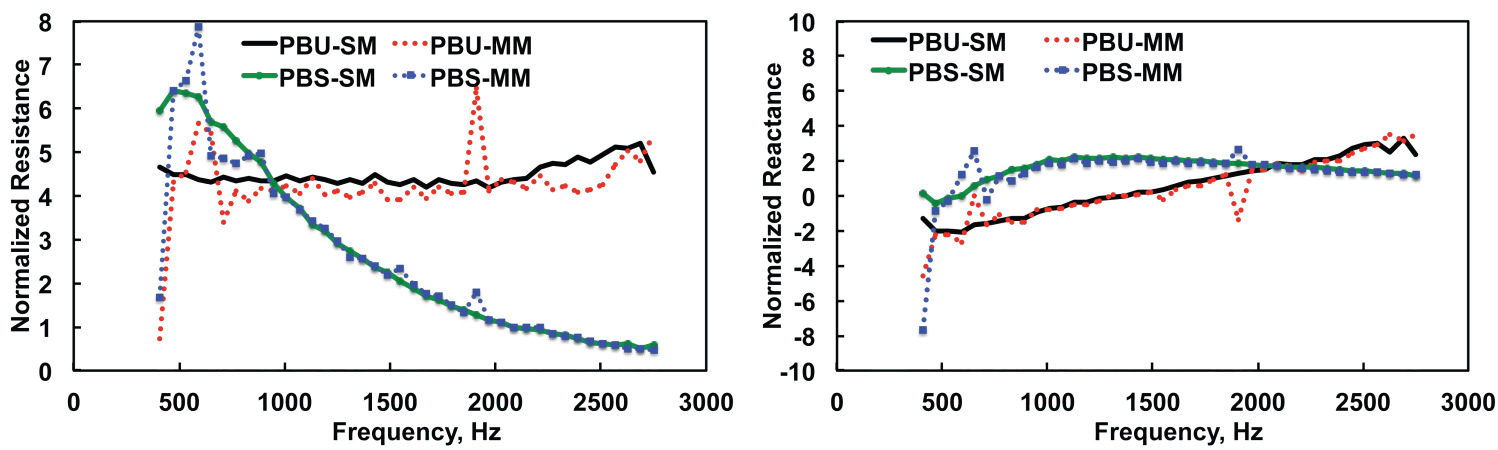

Figure 7: Effects of mean flow profile assumption on educed liner impedance; Mach 0.3, source SPL of $120 \mathrm{~dB}$.

Although the impedance spectra educed with uniform-flow and shear-flow assumptions are distinctly different (see Figs. 6 and 7), each of them provide good fits to the measured acoustic pressures at each of the microphone locations when the corresponding propagation code (i.e., one with the same mean flow assumption) is used. The Pridmore-Brown methods use the measured axial sound pressure level and phase decay rates, whereas the convected Helmholtz method employs minimization of $F(\zeta)$ as the criterion for determining the liner impedance. However, if the impedance eduction method and the propagation code are not properly matched, e.g., if the impedance is educed with a shear-flow method and the propagation code assumes uniform flow (or vice versa), the predicted acoustic pressures at each microphone location should not be expected to compare favorably with the corresponding measured acoustic pressures.

These results highlight the fact that it is important to understand the underlying assumptions of the eduction method that has been used to determine the impedance of a liner. If the educed impedance is to be used as a boundary condition in a propagation code to predict the sound transmission through a duct, it is critical that the impedance boundary condition be consistent with the assumptions used in the propagation code. Further study is needed to better understand just how far this premise should be taken, i.e., to determine when, if ever, it is acceptable to use an impedance educed using a uniform-flow assumption with a propagation code that assumes a shear-flow profile (or vice versa).

The remainder of Section IV presents the effects of mean flow and source SPL (with associated harmonic distortion) on the educed impedance. Each of the current methods (PBU-SM, PBU-MM, PBS-SM, PBS-MM, CHE) are affected similarly by these parameters (e.g., increase in $M$ or SPL causes increase in resistance). As mentioned earlier, all of the data acquired with the current liner appear to be dominated by a single mode. Previous results ${ }^{1}$ demonstrated that a single-mode method is preferable when the measured data are clearly dominated by a single mode, as it provides a direct computation of the impedance (i.e., not an iterative technique) and is therefore very efficient. For these reasons, the remainder of the investigation will concentrate on PBU-SM results.
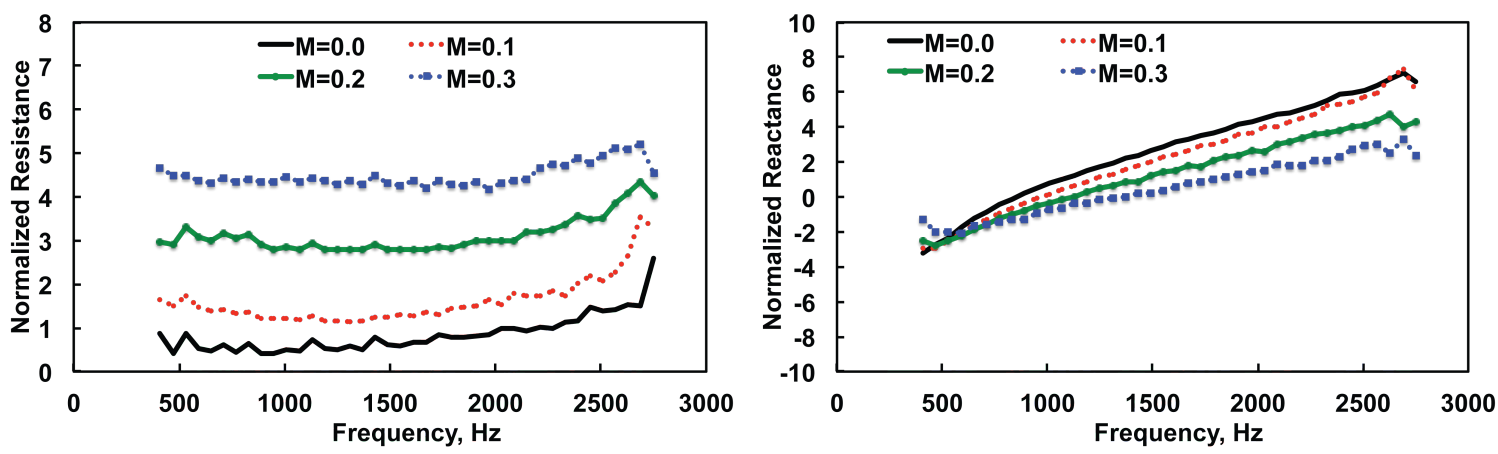

Figure 8: Effects of Mach number on liner impedance; PBU-SM method, source SPL of $120 \mathrm{~dB}$. 

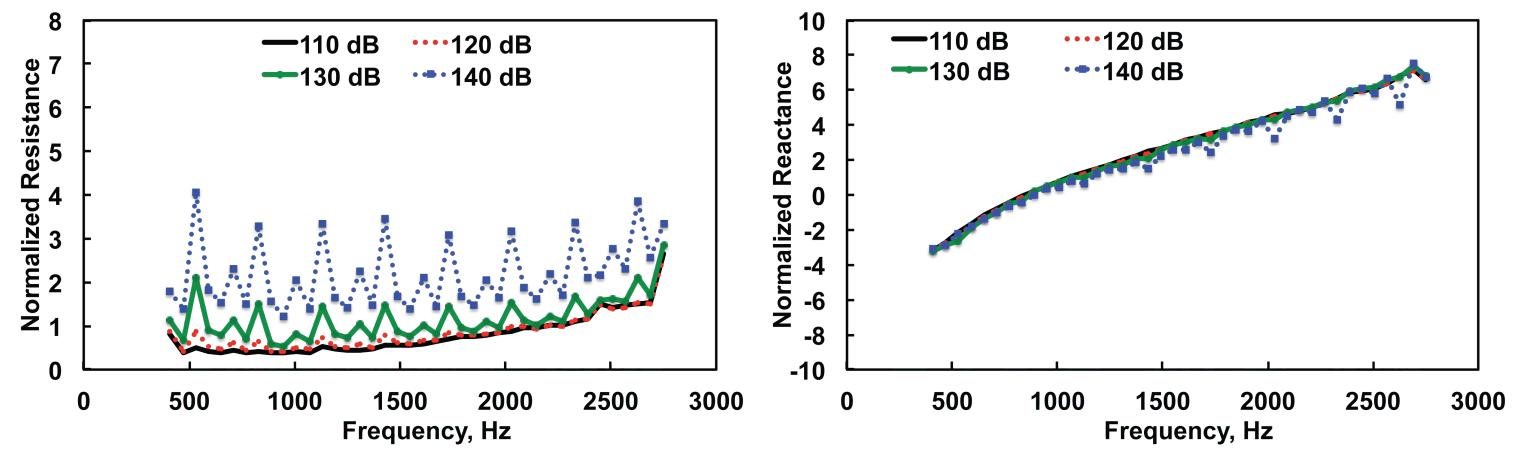

(a) Mach 0.0
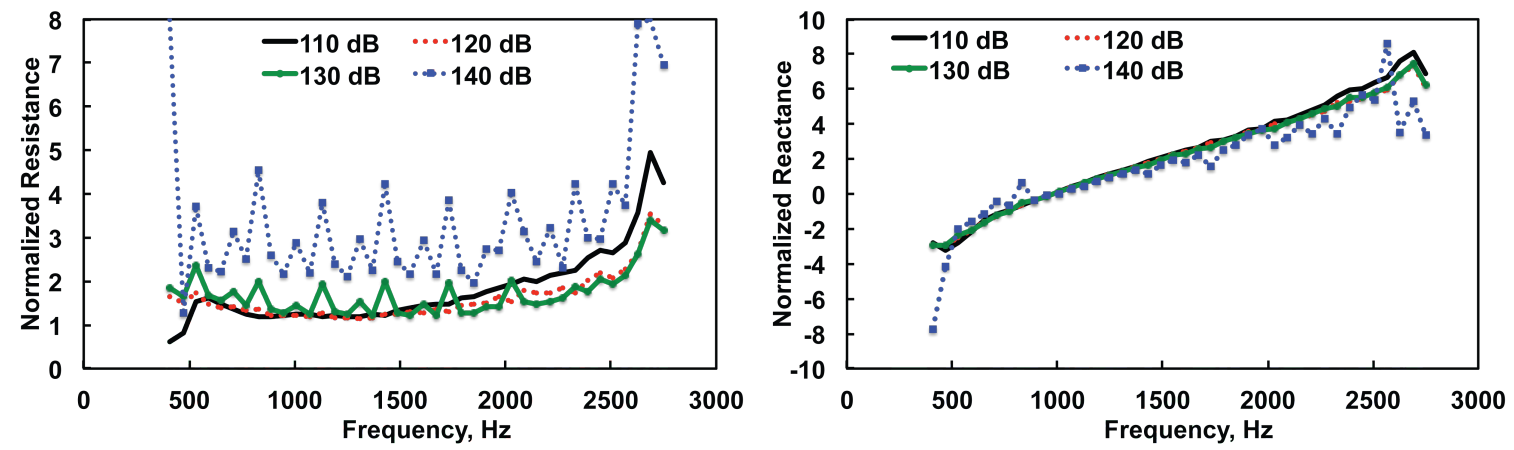

(b) Mach 0.1
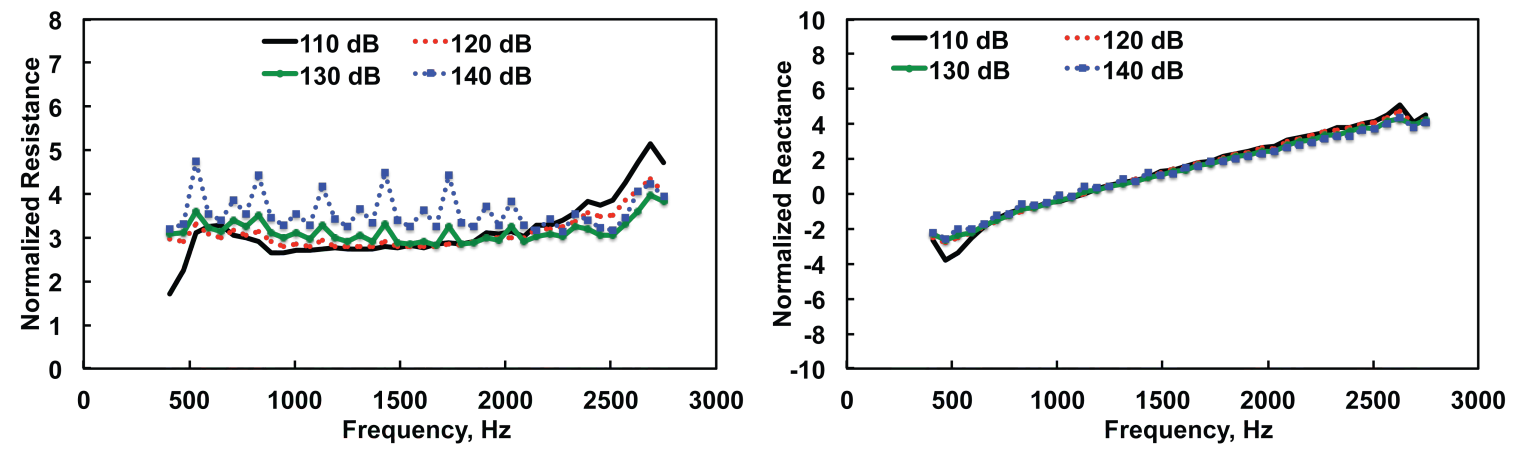

(c) Mach 0.2
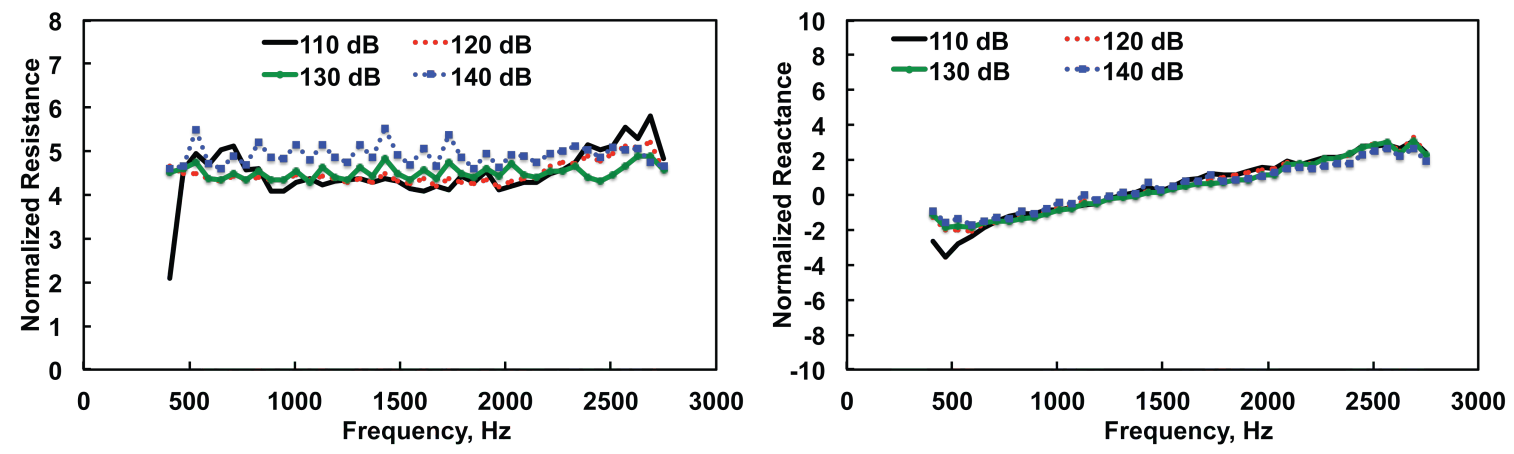

(d) Mach 0.3

Figure 9: Effects of source SPL and harmonic distortion on liner impedance; PBU-SM method, (a) Mach 0.0, (b) Mach 0.1, (c) Mach 0.2, and (d) Mach 0.3. 


\section{B. Effects of Mean Flow on Educed Impedance}

Figure 8 provides a comparison of impedance spectra educed at mean flow Mach numbers of 0.0, 0.1, 0.2 and 0.3 , with a source SPL of $120 \mathrm{~dB}$. As is commonly observed for conventional perforate-over-honeycomb liners, the resistance spectrum is observed to increase with increasing Mach number. The resistance spectra are also observed to contain occasional peaks at selected frequencies (the data points are connected by lines to allow convenient comparisons of the individual spectra). This feature is most noticeable in the Mach 0.0 resistance spectrum, and will be discussed in greater detail below. The reactance spectrum is also observed to flatten with increasing Mach number, as has been demonstrated in previous investigations. ${ }^{13}$

\section{Effects of Source SPL and Harmonic Distortion on Educed Impedance}

Figure 9 provides a comparison of impedance spectra educed at source sound pressure levels of 110, 120, 130 and $140 \mathrm{~dB}$, for centerline Mach numbers of 0.0 to 0.3. Recall that the hole diameter and facesheet thickness are both $1 \mathrm{~mm}$, i.e., the thickness-to-diameter ratio is unity. This thickness-to-diameter ratio is much less than 10, which is often used as a criterion for predicting liner linearity (as this ratio increases, the liner becomes more linear). This suggests the test liner is nonlinear, as confirmed by the increase in resistance as a function of source SPL.

An increase in Mach number or source SPL results in an increase (shift upward) of the resistance spectra. As noted earlier, an increase in Mach number also causes the reactance spectra to decrease in slope (flatten). However, increases in source SPL have very minimal effect on the educed reactance spectra. Figure 9 also provides greater insight into the peakedness of the impedance spectra. These peaks are a dominant feature at Mach 0.0, and become more subdued as the Mach number is increased. Although not nearly as dominant, similar behavior is noted in the corresponding reactance spectra, except the excursions from the expected spectra are generally toward lower values.

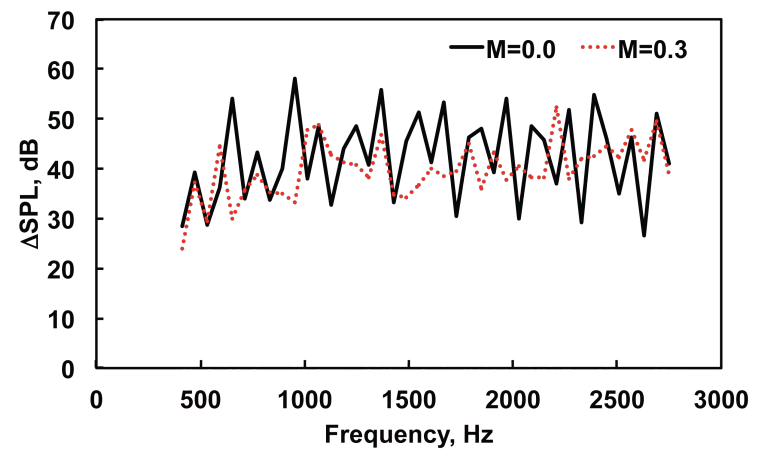

(a) $110 \mathrm{~dB}$

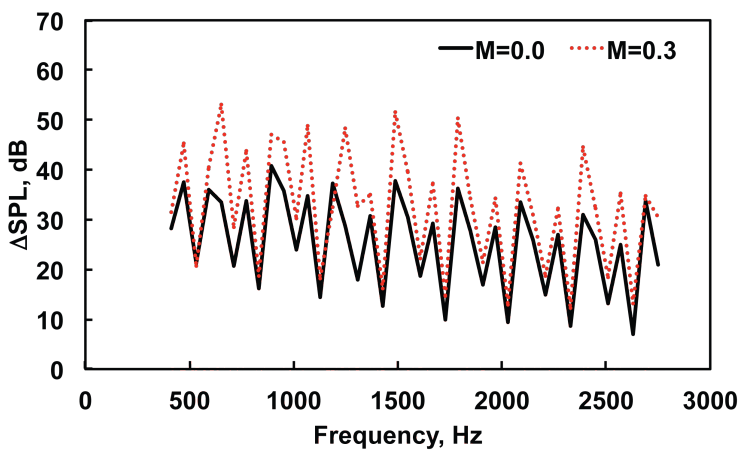

(c) $130 \mathrm{~dB}$

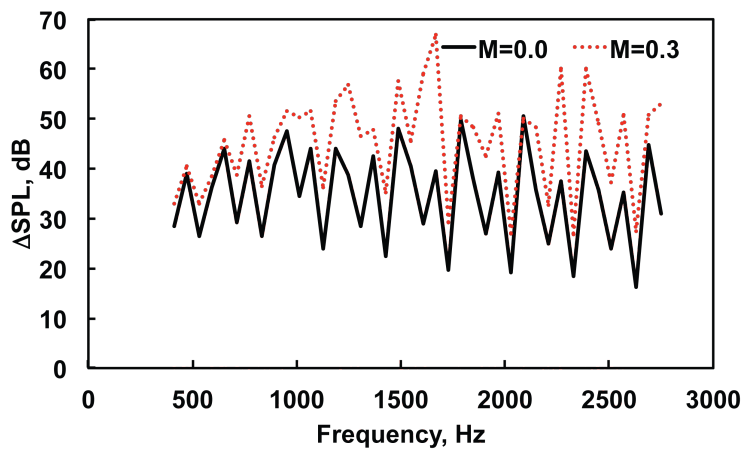

(b) $120 \mathrm{~dB}$

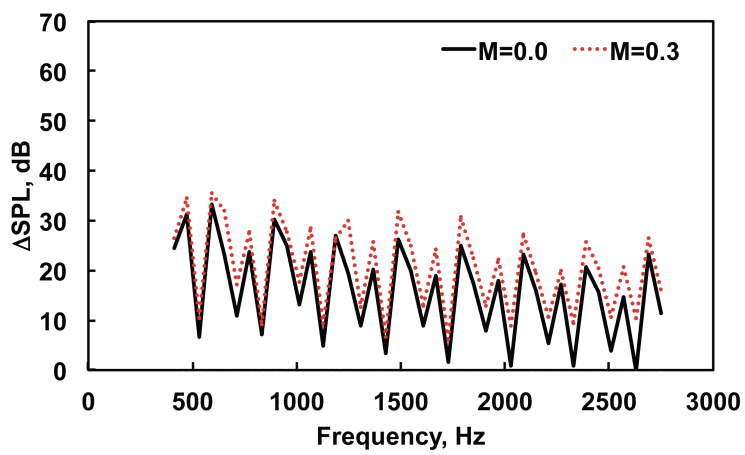

(d) $140 \mathrm{~dB}$

Figure 10: Effects of mean flow on SPL difference between fundamental and first harmonic; source levels for fundamental frequency: (a) $110 \mathrm{~dB}$, (b) $120 \mathrm{~dB}$, (c) $130 \mathrm{~dB}$, (d) $140 \mathrm{~dB}$. 
For each of the Mach numbers included in the test, peaks in the resistance spectra become more pronounced as the source SPL is increased. The linearizing effect of the mean flow is evident in Fig. 9-d, in which the effects of source SPL are greatly reduced. Correspondingly, the peaks in the resistance spectra are observed to be significantly subdued for this case.

The peaks in the resistance spectra appear anomalous. However, an explanation for these resistance peaks (and, to a lesser extent, reactance "dropouts") is found in the results depicted in Fig. 10. This figure presents the differences between the fundamental and first harmonic SPLs, as measured with the reference microphone (at $x=0$, see Fig. 1). As mentioned in Section III, the electrical signal input to the acoustic drivers consists of a pure tone at the selected frequency. However, the acoustic drivers are nonlinear devices. Thus, if the input signal is a pure tone at frequency $f$, an additional tone is generated at $2 f$ when the amplitude of the input signal becomes sufficiently large. The acoustic drivers are operating well within their linear regime at the lower source SPL $(110 \mathrm{~dB}$, Fig. 10-a), such that the SPL of the first harmonic $(2 f)$ is at least $20 \mathrm{~dB}$ below that of the fundamental $(f)$ across the entire frequency range of interest. The difference between the two SPLs (fundamental minus first harmonic) decreases as the source SPL is increased, and approaches zero for a number of frequencies as the source level is increased to $140 \mathrm{~dB}$ (Fig. 10-d). At a source level of $140 \mathrm{~dB}$, the acoustic drivers are being pushed well into their nonlinear regime.

Careful comparison of Figs. 9 and 10 reveals a direct correlation between the presence of high harmonic levels (i.e., small differences between fundamental and first harmonic) and peaks in the resistance spectra. The magnitude of the resistance peaks is observed to increase as the first harmonic SPL approaches that of the fundamental. Clearly, the acoustic particle velocity at frequencies other than the fundamental (in this case, the first harmonic) has a direct effect on the impedance observed at the fundamental. This effect is often incorporated into impedance prediction models via an RMS acoustic particle velocity term. For example, the Two-Parameter Model ${ }^{14,15}$ computes the normalized acoustic resistance as $\theta=A+B v_{r m s}$, where $A$ and $B$ are semi-empirical constants based on the liner geometry and the local aeroacoustic environment, and $v_{r m s}$ is the RMS acoustic particle velocity measured across the entire frequency range of interest. However, impedance prediction models do not typically include a similar term for the acoustic reactance component. Although the effects of high harmonic levels are much less evident in the measured reactance spectra, they become clearly evident at the highest source SPL $(140 \mathrm{~dB})$. These results suggest that a coupling term (e.g., $\left.v_{r m s}\right)$ should also be present in the acoustic reactance prediction.

These results demonstrate the fact that the impedance of a conventional, perforate-over-honeycomb liner is a function of the aeroacoustic environment that is present at the surface of the liner. Thus, an impedance spectrum educed in the presence of broadband noise should be expected to be different from an impedance spectrum educed with a single-tone source (one frequency at a time). Further, any combination of sources (e.g., broadband plus tone or multiple tones) should be expected to yield yet another impedance spectrum. This does not mean that any of these independent impedance spectra are incorrect. Rather, it highlights the importance of using the impedance spectrum that has been educed with a source that is similar to the one to be used in the final application, i.e., if the final application will have a broadband sound source, then the appropriate impedance spectrum to be used in the aeroacoustic propagation code is one that is educed using a similar broadband sound source.

In summary, the outliers in the impedance spectra provided in Fig. 9 are not anomalous values, but are simply due to the presence of significant harmonic content in the sound source used in the acoustic liner test. As such, they correctly represent the impedance of the acoustic liner when exposed to this particular aeroacoustic environment. However, if the purpose of the test is to determine the impedance of the acoustic liner when exposed to single tones (i.e., no harmonic content), further evaluation of the data is required. As seen in Fig. 9, the effects of the harmonic content become less important as the flow Mach number is increased. It is expected that these effects are also a function of the liner geometry parameters (e.g., hole diameter, facesheet thickness, porosity), and may not necessarily be as dramatic for other liners as for the one used in this test. Nevertheless, it is important to remain cognizant of the potential effects of harmonic content. It is also important to note that the current analyses assume linear acoustic propagation, and do not account for wave steepening effects that occur at sufficiently high source sound pressure levels.

An investigation is conducted to quantify the effects of harmonic distortion on the educed liner impedance, such that the corresponding single-tone (no harmonic content) impedance spectra can be "extracted." This investigation is based on the assumption that the resistance spectrum should be a smooth function of frequency. Figure 11 provides a demonstration as to how this process is performed. The acoustic resistance 
spectrum educed from measured data is depicted with blue squares, and the "corrected" resistance spectrum is depicted with red circles. The blue squares are covered up with the red circles at the majority of the test frequencies. Essentially, a smoothing process is applied to the measured spectrum to achieve the corrected spectrum. This smoothing process consists of removing resistance values at frequencies for which the difference between the fundamental and first harmonic SPLs is less than a selected value, $\Delta_{s}$. This smoothing parameter, $\Delta_{s}$, is gradually increased (i.e., additional values that are assumed anomalous due to excessive harmonic content are removed) until the resultant resistance spectrum is a smooth function of frequency.

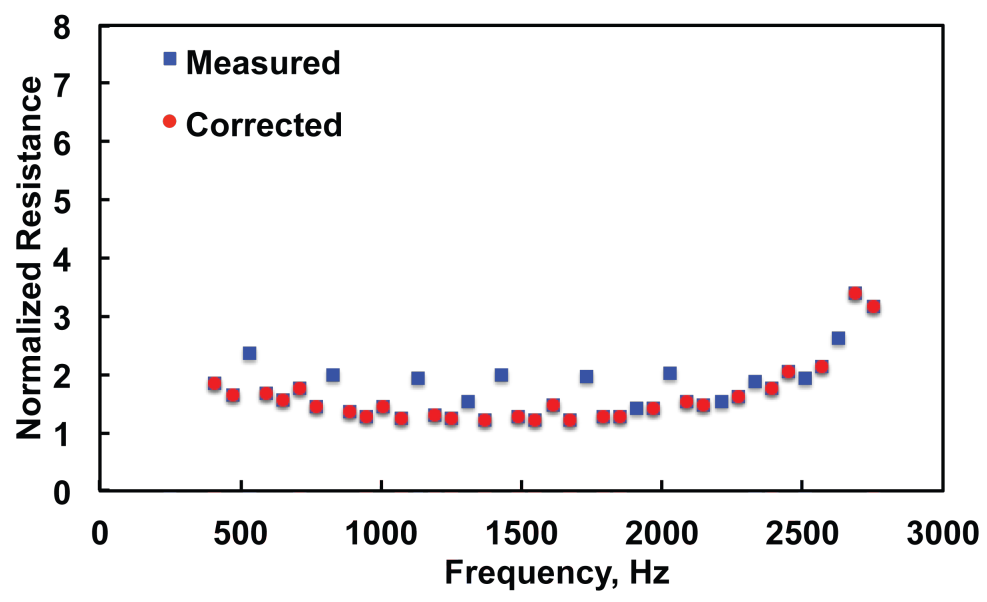

Figure 11: Demonstration of process to eliminate effects of harmonic distortion on liner impedance; PBU-SM method, source SPL of $130 \mathrm{~dB}$, Mach 0.1 .

In the example shown in Fig. 11, $\Delta_{s}$ is set at $20 \mathrm{~dB}$, i.e., red squares represent resistance values educed at frequencies for which the SPL at the first harmonic is at least $20 \mathrm{~dB}$ below that of the fundamental. As expected, based on the results of Figs. 9 and 10, the smoothing parameter is dependent on the source SPL and the mean flow Mach number. As the source SPL is increased, the acoustic drivers become more nonlinear and thus generate increased harmonic content. However, as the mean flow Mach number is increased, this effect becomes more subdued. The data educed in this investigation indicate two potential causes for this latter result. An increase in mean flow Mach number linearizes the acoustic impedance. A Mach number increase also results in increased flow noise that tends to mask the effect of the harmonic distortion.

This smoothing process is performed for each combination of source SPL and mean flow Mach number, as follows:

1. The source acoustic pressure magnitude, $P_{f}$, at the fundamental frequency is determined as $P_{f}=P_{r e f} 10^{\mathrm{SPL}_{\mathrm{f}} / 20}$ where $\mathrm{SPL}_{\mathrm{f}}$ is the SPL at the fundamental frequency and $P_{r e f}$ is the reference pressure, $20 \mu \mathrm{Pa}$.

2. The value of $\Delta_{s}=\mathrm{SPL}_{\mathrm{f}}-\mathrm{SPL}_{\mathrm{h}}$ needed to achieve a smooth acoustic resistance spectrum is determined.

3. The source acoustic pressure, $P_{h}$, at the first harmonic frequency for which a smooth acoustic resistance spectrum is achieved is determined as $P_{h}=P_{r e f} 10^{\Delta_{s}} / 20$.

4. Steps 1-3 are performed for each combination of source SPL and mean flow Mach number, resulting in $P_{f}(\mathrm{SPL}, \mathrm{M})$ and $P_{h}(\mathrm{SPL}, \mathrm{M})$.

5. For each Mach number, the results at each $\mathrm{SPL}_{\mathrm{f}}$ are curve-fit using $\frac{P_{f}}{P_{h}}=\alpha e^{-\beta P_{f}}$.

6. Corresponding values of $\Delta_{s}$ across the entire $\mathrm{SPL}_{\mathrm{f}}$ range of interest are computed as

$$
\Delta_{s}\left(\mathrm{SPL}_{\mathrm{f}}\right)=\mathrm{SPL}_{\mathrm{f}}-\mathrm{SPL}_{\mathrm{h}}=20 \log _{10}\left(\frac{P_{f}}{P_{h}}\right)
$$




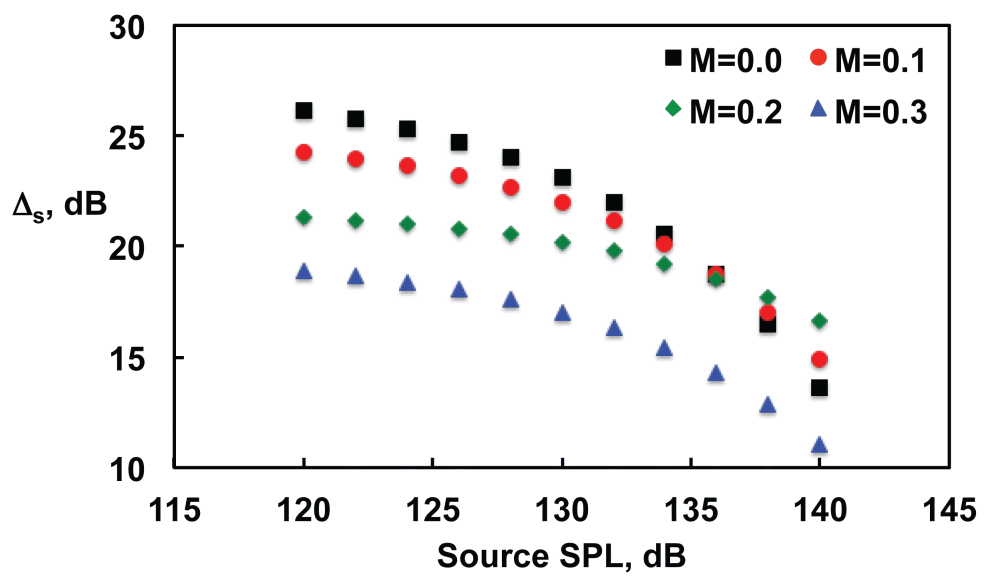

Figure 12: Sound pressure level difference $\left(\Delta_{s}=\mathrm{SPL}_{\mathrm{f}}-\mathrm{SPL}\right)$ for which smooth acoustic resistance spectra are achieved.

Figure 12 provides a comparison of the resultant curves computed for each of the four Mach numbers used in the current study. In spite of the heuristic approach used to determine $\Delta_{s}(\mathrm{SPL}, M)$, the four curves in Fig. 12 are well behaved. The SPL difference, $\Delta_{s}(\mathrm{SPL}, M)$, for which a smooth acoustic resistance spectrum is achieved generally decreases with increasing Mach number and/or increasing source SPL. This last point is of particular interest. Earlier, it was noted that peaks in the acoustic resistance spectra grow as the source SPL is increased. The results of Fig. 12 indicate that this occurs because the difference between the fundamental and first harmonic SPLs is decreasing in a disproportionate manner relative to the increase in source SPL. In other words, an increase in source SPL only causes narrow-band spikes in the educed acoustic resistance when that increase in source SPL is accompanied by a corresponding increase in harmonic distortion (i.e., when first harmonic level approaches that of the fundamental).

\section{Concluding Remarks}

This investigation has explored the use of multiple impedance eduction methods to study the acoustic performance of a single-layer, perforate-over-honeycomb liner. This liner was fabricated by the German Aerospace Center and tested in the NASA Langley Grazing Flow Impedance Tube. A number of conclusions can be drawn from the data presented in this investigation.

1. Axial wavenumbers educed using the Prony method have been incorporated into an impedance eduction method based on the Pridmore-Brown equation, thereby allowing this method to account for the presence of multiple modes in the lined region of the GFIT.

2. Each of the impedance eduction methods that assume uniform flow (PBU-SM, PBU-MM, CHE) produce impedance spectra that compare favorably over the majority of the frequency range used in this investigation (410 to $2750 \mathrm{~Hz}$ ). Deviations at the upper frequency extreme are due to the onset of higher-order modes. At the lower frequency extreme, deviations are possibly due to the fact that the liner length is a fraction of the test frequency wavelength. This supposition needs further exploration.

3. Each of the impedance eduction methods that assume shear flow (PBS-SM, PBS-MM) produce impedance spectra that compare favorably, except at the lowest frequencies. Again, this is likely due to the increased wavelengths at these frequencies. However, this supposition also needs further exploration.

4. As the mean flow Mach number is increased, the resistance spectra educed with the uniform-flow, single-mode method (PBU-SM) shift upward (higher values) and the reactance spectra become flatter 
(approach zero slope). Given the thickness-to-diameter ratio (unity) of the perforates in the facesheet, this result is expected.

5. As the source sound pressure level is increased, the resistance spectra educed with the PBU-SM shift upward. However, increases in the SPL have virtually no effect on the corresponding reactance spectra.

6. Harmonic distortion effects must be taken into account in the impedance eduction process. Results presented with the PBU-SM method (also observed with each of the other methods considered) clearly demonstrate that the resistance educed at a single frequency is increased if the harmonic content is sufficiently high. Deviations from the expected values are also observed in the educed reactance, but are much less significant than those observed in the resistance. This coupling effect is captured in conventional liner impedance prediction models for the resistance component, but is not typically included for the reactance component. The current results suggest a coupling term should be included for the reactance component.

7. The difference between the SPLs of the fundamental and first harmonic frequencies that is required to inhibit this coupling effect depends on the mean flow Mach number and source SPL used in the test. An approach was presented to provide guidelines regarding how to determine the impedance spectra that would be expected if the harmonic distortion were eliminated.

8. The impedance of a conventional, perforate-over-honeycomb liner is a function of the acoustic source that is present at the liner surface. Ideally, the impedance boundary condition used in an aeroacoustic propagation code should be representative of the sound field that will be present in the desired application. If this cannot be achieved, this may cause the sound absorption of the liner to be incorrectly predicted.

Clearly, some of these conclusions (e.g., items 4 and 5) are specific to the liner considered in this investigation. Otherwise, the results of this investigation provide valuable insight regarding the results achieved with the distinct impedance eduction methods, and also provide useful guidance regarding usage of data for which significant harmonic content is present.

\section{Acknowledgements}

The authors wish to express their appreciation to Carl Gerhold and Chris Jasinski for their support in the implementation of the multi-mode impedance eduction method. This research was funded by the Fixed Wing Project of NASA's Fundamental Aeronautics Program.

\section{References}

${ }^{1}$ Jones, M. G., Watson, W. R., Tracy, M. B., and Parrott, T. L., "Comparison of Two Waveguide Methods for Educing Liner Impedances in Grazing Flow," AIAA Journal, Vol. 42, No. 2, Februrary 2004, pp. 232-240.

${ }^{2}$ Watson, W. R. and Jones, M. G., "Comparison of Convected Helmholtz and Euler Model for Impedance Eduction in Flow," AIAA Paper 2006-2643, May 2006.

${ }^{3}$ Jones, M. G., Parrott, T. L., and Watson, W. R., "Comparison of Acoustic Impedance Eduction Techniques for LocallyReacting Liners," AIAA Paper 2003-3306, May 2003.

${ }^{4}$ Syed, A. A., Yu, J., Kwan, H. W., and Chien, E., "The Steady Flow Resistance of Perforated Sheet Materials in High Speed Grazing Flows," NASA CR 2002-211749, 2002.

${ }^{5}$ Gallman, J. M. and Kunze, R. K., "Grazing Flow Acoustic Impedance Testing for the NASA AST Program," AIAA Paper 2002-2447, June 2002.

${ }^{6}$ Busse-Gerstengarbe, S., Bake, F., Enghardt, L., and Jones, M. G., "Comparative Study of Impedance Eduction Methods, Part 1: DLR Tests and Methodology," 19th AIAA/CEAS Aeroacoustics Conference, American Institute of Aeronautics and Astronautics, Reston, VA (submitted for publication).

${ }^{7}$ Pridmore-Brown, D. C., "Sound Propagation in a Fluid Flowing Through an Attenuating Duct," Journal of Fluid Mechanics, Vol. 4, 1958, pp. 393-406.

${ }^{8}$ Myers, M. K., "On the Acoustic Boundary Condition in the Presence of Flow," Journal of Sound and Vibration, Vol. 71, No. 3, 1980, pp. 429-434.

${ }^{9}$ Marple, S. L., Digital Spectral Analysis with Applications, Prentice-Hall, 1987.

${ }^{10}$ Watson, W. R. and Jones, M. G., "Impedance Eduction in Ducts with Higher-Order Modes and Flow," AIAA Paper 2009-3236, May 2009. 
${ }^{11}$ Bendat, J. S. and Piersol, A. G., Random Data: Analysis and Measurement Procedures, Wiley-Interscience, 1971.

${ }^{12}$ Watson, W. R. and Jones, M. G., "Evaluation of Wall Boundary Conditions for Impedance Eduction Using a Dual-Source Method," AIAA Paper 2012-2199, June 2012.

${ }^{13}$ Watson, W. R. and Jones, M. G., "Validation of a New Procedure for Impedance Eduction in Flow," AIAA Paper 2010-3764, May 2010.

${ }^{14}$ Motsinger, R. E. and Kraft, R. E., "Design and Performance of Duct Acoustic Treatment: Aeroacoustics of Flight Vehicles; Chapter 14, Vol. 2: Noise Control," NASA RP 1258, August 1991.

${ }^{15}$ Parrott, T. L. and Jones, M. G., "Assessment of NASA's Aircraft Noise Prediction Capability, Chapter 6: Uncertainty in Acoustic Liner Impedance Measurement and Prediction," NASA TP 2012-215653, July 2012. 